2006-01-01

\title{
Amplification and suppression of internal waves by tides over variable bottom topography
}

\section{Vlasenko, Vasyl}

http://hdl.handle.net/10026.1/3856

\subsection{5/jpo2958.1}

Journal of Physical Oceanography

American Meteorological Society

All content in PEARL is protected by copyright law. Author manuscripts are made available in accordance with publisher policies. Please cite only the published version using the details provided on the item record or document. In the absence of an open licence (e.g. Creative Commons), permissions for further reuse of content should be sought from the publisher or author. 


\title{
Amplification and Suppression of Internal Waves by Tides over Variable Bottom Topography
}

\author{
Vasiliy Vlasenko and Nataliya Stashchuk \\ School of Earth, Ocean and Environmental Sciences, University of Plymouth, Plymouth, United Kingdom
}

(Manuscript received 21 March 2005, in final form 20 March 2006)

\begin{abstract}
The energy exchange between internal waves and barotropic currents over inclined bottom topography is studied theoretically and in the framework of the numerical model. The energy balance equation derived for a continuously stratified fluid predicts that energy can either be transferred toward or away from the internal wave depending on the direction of propagation of both the wave and current. Four scenarios of wave-flow interaction over the inclined bottom were identified. An internal wave extracts energy from the background tidal flow during its propagation upslope-upstream or downslope-downstream and its amplitude grows. The wave loses energy propagating downslope-upstream or upslope-downstream and reduces in amplitude. This mechanism of suppression or amplification of internal waves by a current over an inclined bottom is verified numerically. When applied to the area of the Knight Inlet sill, a high-resolution fully nonlinear, nonhydrostatic model reproduces the packets of internal waves generated by supercritical tidal flow over the sill. Careful inspection of the wave fields revealed the presence of an irregular wave structure within wave packets-namely, internal waves are not arranged by amplitude. This phenomenon, obtained numerically and observed in situ, is treated in terms of the mechanism of wave-flow interaction: the energy exchange between the tidal current and generated internal waves over the inclined bottom topography is the reason for the absence of traditional rank-ordered waves in the packet.
\end{abstract}

\section{Introduction}

The problem of energy exchange between internal waves and currents is described in many publications, for example, in Turner (1973), LeBlond and Mysak (1979), and Baines (1995) (see also related references in these books). In the simplest case when linear periodic internal waves interact with a shear current, the latter modifies the waveform and wave speed when it enters the critical layer where phase speed is equal to current velocity. After reflection from the critical layer, the wave restores its form and phase speed to their original values. As a result there is no net exchange of energy and momentum between linear waves and shear currents. However, in many oceanic observations the internal waves were found to be sufficiently nonlinear having the form of internal bores, or solitary waves. In

Corresponding author address: Vasiliy Vlasenko, School of Earth, Ocean and Environmental Sciences, University of Plymouth, Plymouth PL4 8AA, United Kingdom.

E-mail: vvlasenko@plymouth.ac.uk such a case an interaction of nonlinear internal waves with the background currents becomes more complicated.

In a weakly nonlinear case, the Korteweg-de Vries theory can be used for the analysis of wave-flow interaction. It has been modified in many ways to take into account the effects on waves of steady shear currents (Lee and Beardsley 1974; Maslowe and Redekopp 1980; Clarke and Grimshaw 1999), irregular bottom topography (Djordjevic and Redekopp 1978), currents variable with time (Zhou and Grimshaw 1989), or position of the pycnocline varying together with the bottom topography (e.g., Pelinovsky et al. 1995). The effect of vertical shear of currents on internal waves was only considered in such an approach.

Horn et al. (2000) expanded the weakly nonlinear theory to show wave evolution in a two-layer fluid in the presence of a strong space-time varying background flow. Irregular bottom topography was also included in the analysis. The theory was developed for investigation of lake dynamics, namely, for the study of the disintegration of a basin-scale internal wave (baroclinic seiche) into a series of internal solitary waves 
(ISWs). In this problem the background flow had current-countercurrent structure with strong shear in the density interface layer. Introducing the concept of "local energy" calculated as an integral of the interface displacement, it was shown that the wave amplitude in the packet is locally either increased or decreased as it propagates through a space-time-varying background field. Having the rank-ordered wave packet, Horn et al. (2000) did clarify the possibility of the energy exchange between the current and the solitary waves.

The idea of the energy transfer from the background flow to baroclinic solibore (sawtooth-shaped waves in a train instead of the sinusoidal form) was also used by Henyey and Hoering (1997), who similar to Long (1970) developed the energy balance equation for a two-layer fluid and estimated the energy flux into the propagating wave train. It was shown that the nonzero energy flux into the internal wave train can exist because of the different stratification in front of and behind the propagating solibore (similar to a surface bore). Note, however, that the accuracy of a control volume approach in a two-layer fluid is quite poor (up to $100 \%$; see Cummins and $\mathrm{Li} \mathrm{1998)} \mathrm{and} \mathrm{application} \mathrm{of}$ this method is restricted by the correct choice of jump relation. The next limitation of this method is that it can only be used for the waves propagating in a basin of constant depth.

Unlike Henyey and Hoering (1997) who assumed that the energy flux to the wave train is exactly compensated by the energy sink due to the dissipation, the goal of the present study is to investigate the essentially time dependent processes of the growth and decay of internal waves by tidal current over inclined bottom topography. The barotropic flow basically has strong horizontal shear in addition to the traditionally investigated vertical shear. The energy balance equation is used as a tool for the analysis of wave-flow interaction.

The paper is organized as follows. The energy balance equation for internal waves propagating on the background of a barotropic current over inclined bottom topography is derived in section 2. The model study on the amplification and suppression of internal waves by a barotropic current in a shelf-slope area is carried out in section 3. Section 4 presents the discussion of how the derived energy balance equation can be used for the explanation of the effect of non-rankordering in packets of tidally generated ISWs. The area of the Knight Inlet sill is considered as a case study. A summary and conclusions are presented in the final section.

\section{Energy balance equation}

Consider now internal waves propagating on the background of a barotropic flow. The study addresses the problem of understanding the mechanism of waveflow interaction over irregular bottom topography. The analysis of the energy balance equation in such a system can clarify the possibility of energy transfer from currents to waves and vice versa. For its formulation we introduce the right-handed Cartesian coordinate system, in which the $x$ axis is directed perpendicular to the isobaths, the $y$ axis is along the bathymetry, and the $z$ axis is directed vertically upward; $z=0$ corresponds to the free surface in a steady state. The bottom topography is taken to be a function of $x$ only, $H=H(x)$.

We assume that the wave field over irregular bottom topography can be decomposed into the background barotropic current and baroclinic wave perturbations. In doing so, we introduce the velocity vector $\mathbf{V}=\{U, W\}$, pressure $P$, and density $\bar{\rho}$ for a stationary barotropic current, and $\mathbf{v}=\{u, w\}, p$, and $\rho$ for the wave perturbation, respectively. Here $U, u$ are the velocity in the $x$ direction, and $W, w$ are the vertical components. We restrict our analysis to the consideration of a stationary background current. However, for tidally generated ISWs the barotropic tidal flow, which is time-dependent $\sim \sin (\omega t)$, where $\omega$ is the tidal frequency, can be considered as a good approximation of a stationary flow because of the large difference between the temporal scales of ISWs (several minutes) and the period of a semidiurnal tide.

For the weak baroclinic motions, that is, when $\left|\mathbf{v}_{t}\right| \gg$ $|\mathbf{v} \cdot \nabla \mathbf{v}|$ and $\left|\rho_{t}\right| \gg|\mathbf{v} \cdot \nabla \rho|$, the following governing system is valid (here the Boussinesque approximation is used):

$$
\begin{gathered}
\bar{\rho}_{0} \frac{\partial \mathbf{v}}{\partial t}+\bar{\rho}_{0}(\mathbf{V} \cdot \boldsymbol{\nabla}) \mathbf{v}+\bar{\rho}_{0}(\mathbf{v} \cdot \boldsymbol{\nabla}) \mathbf{V}+\boldsymbol{\nabla} p+g \rho \mathbf{k}=-\left[\bar{\rho}_{0}(\mathbf{V} \cdot \boldsymbol{\nabla}) \mathbf{V}+\boldsymbol{\nabla} P+g \bar{\rho}_{0} \mathbf{k}\right] \quad \text { and } \\
\frac{\partial \rho}{\partial t}+(\mathbf{V} \cdot \boldsymbol{\nabla}) \rho+(\mathbf{v} \cdot \boldsymbol{\nabla}) \bar{\rho}=-(\mathbf{V} \cdot \boldsymbol{\nabla}) \bar{\rho}, \quad \boldsymbol{\nabla} \cdot \mathbf{v}=-\boldsymbol{\nabla} \cdot \mathbf{V},
\end{gathered}
$$

where $\mathbf{k}$ is the vertical unity vector, $\bar{\rho}_{0}$ is a constant average value of density, and $g$ is the acceleration due to gravity. The right-hand side of system (1) represents the background current, whereas its left-hand side de- 
scribes the wave perturbations. It is evident that system (1) must be valid in the absence of baroclinic motions. If so, it splits into two systems:

1) for the background current

$$
\begin{aligned}
\bar{\rho}_{0}(\mathbf{V} \cdot \boldsymbol{\nabla}) \mathbf{V}+\boldsymbol{\nabla} P+g \bar{\rho}_{0} \mathbf{k} & =0, \\
(\mathbf{V} \cdot \boldsymbol{\nabla}) \bar{\rho} & =0, \quad \text { and } \\
\boldsymbol{\nabla} \cdot \mathbf{V} & =0 ;
\end{aligned}
$$

2) for the wave motions

$$
\begin{array}{r}
\bar{\rho}_{0} \frac{\partial \mathbf{v}}{\partial t}+\bar{\rho}_{0}(\mathbf{V} \cdot \boldsymbol{\nabla}) \mathbf{v}+\bar{\rho}_{0}(\mathbf{v} \cdot \boldsymbol{\nabla}) \mathbf{V}+\nabla p+g \rho \mathbf{k}=0, \\
\frac{\partial \rho}{\partial t}+(\mathbf{V} \cdot \boldsymbol{\nabla}) \rho+(\mathbf{v} \cdot \boldsymbol{\nabla}) \bar{\rho}=0,
\end{array}
$$

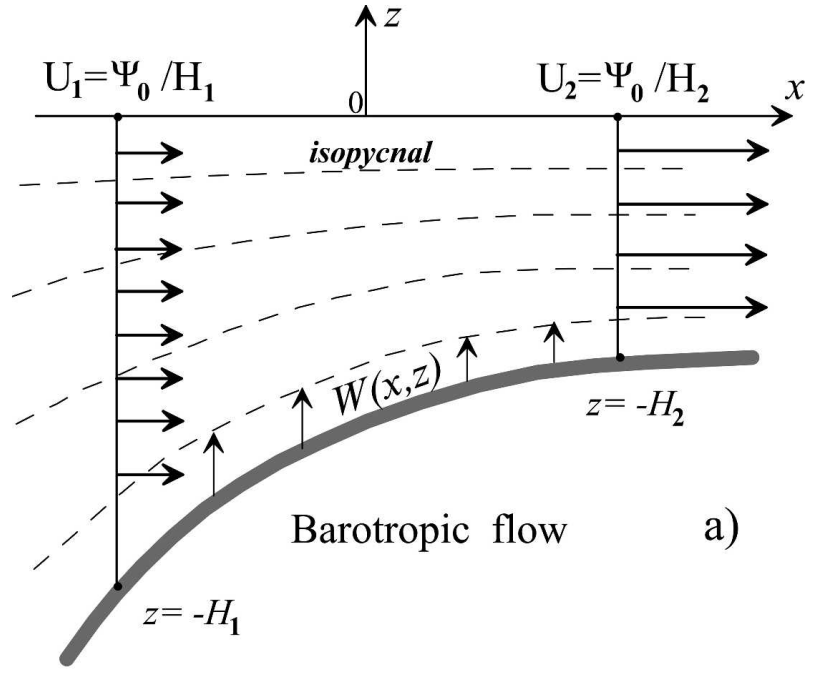

System (2) describes a stationary background current over variable bottom topography. The solution of system (2) can be found numerically in a very general nonlinear case. However, for the exponential bottom topography

$$
H(x)=H_{0} \exp (-\gamma x)
$$

where $H_{0}$ and $\gamma$ are arbitrary constants, the first and third equations in system (2) have the following analytical solution:

$$
U(x, z)=\frac{\Psi_{0}}{H(x)} \quad \text { and } \quad W(x, z)=-\Psi_{0} z \frac{\partial}{\partial x}\left[\frac{1}{H(x)}\right],
$$

where $\Psi_{0}=$ const is the flow discharge, which is also an arbitrary value.

Introducing the streamfunction $\Psi(U=\partial \Psi / \partial z, W=$ $-\partial \Psi / \partial x)$ the second equation in system (2) can be rewritten as $\bar{\rho}_{x} \Psi_{z}-\bar{\rho}_{z} \Psi_{x}=0$, or, alternatively, $J(\bar{\rho}, \Psi)=0$. The last condition means that the density $\bar{\rho}$ is expressed as an arbitrary function of $\Psi$, that is, $\bar{\rho}=\bar{\rho}(\Psi)$. In other words, if a barotropic tidal flux (5) streamlines the bottom topography in a continuously stratified fluid, isopycnals must follow the streamlines (see Fig. 1a).

Thus, in addition to the traditional vertical oceanic stratification, a stationary horizontal density gradient must also exist over an inclined bottom in the case of a steady current. Note that this horizontal gradient is several orders weaker than its vertical counterpart (bottom inclination normally does not exceed several degrees), which is why the terms with horizontal density gradients can be neglected if they appear with perturbation velocities.

The wave density perturbations can be presented in

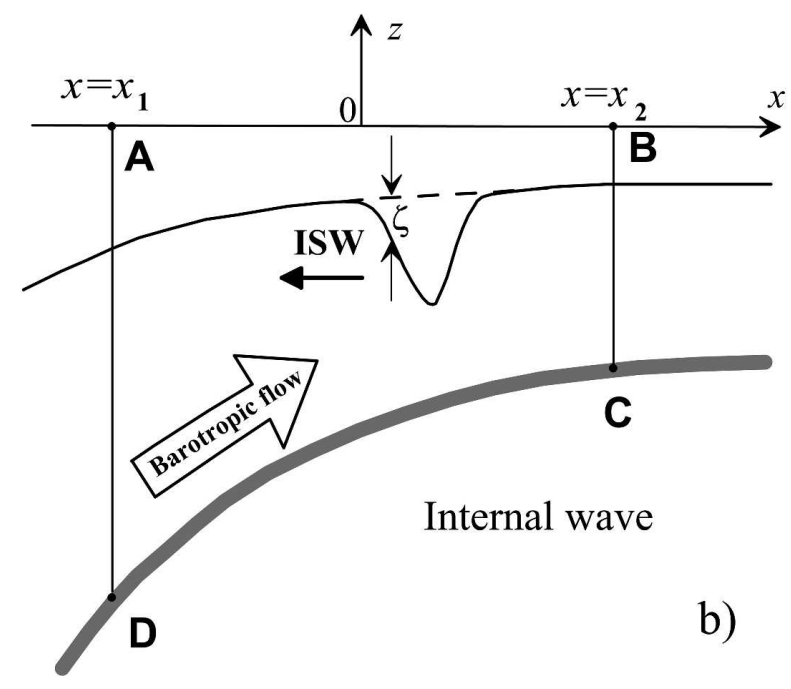

FIG. 1. (a) Schematic diagram presenting the barotropic flux streamlining the bottom topography. (b) Scheme of an internal solitary wave propagating in a basin of variable depth; $\zeta$ is the isopycnal displacement from the equilibrium position.

terms of the Taylor series $\rho(x, z, t)=-\bar{\rho}_{z}(x, z) \zeta(x, z, t)+$ $O\left(\zeta^{2}\right)$. Here the vertical isopycnal displacement $\zeta$ is introduced as presented in Fig. 1b. With accuracy $O\left(\zeta^{2}\right)$ the density can be rewritten as follows: $\rho(x, z, t)=$ $N^{2} \zeta \bar{\rho} / g$, where $N=\left(-g \bar{\rho}_{z} / \bar{\rho}_{0}\right)^{1 / 2}$ is the buoyancy frequency.

Multiplying the momentum balance equation in (3) by $\mathbf{v}$, system (3) is transformed into the energy balance equation

$$
\frac{\partial E}{\partial t}+\nabla \mathbf{F}=-R,
$$

where the density of energy $E$, the energy flux $\mathbf{F}$, and Reynolds stresses $R$ are expressed as follows: 


$$
\begin{aligned}
E= & \bar{\rho}_{0}\left(\mathbf{v}^{2}+N^{2} \zeta^{2}\right) / 2, \\
\mathbf{F}= & \mathbf{V} E+\mathbf{v} p, \text { and } \\
R= & \bar{\rho}_{0}\left[u^{2} U_{x}+w^{2} W_{z}+u w\left(W_{x}+U_{z}\right)\right. \\
& \left.+\zeta^{2} g / \bar{\rho}_{0}\left(\bar{\rho}_{x} U_{z}-\bar{\rho}_{z} U_{x}\right) / 2\right] .
\end{aligned}
$$

The term $R$ in the right-hand side of (6) represents the wave-flow interaction. In the case of barotropic flux over an inclined bottom this term includes not only traditionally considered vertical shears $U_{z}$ and $W_{z}$, but also horizontal shears $U_{x}$ and $W_{x}$. Taking into account the analytical solution (5) for an exponential bottom profile (4), (9) can be simplified as follows:

$$
R=\bar{\rho}_{0} \Psi_{0}\left\{\left(u^{2}-w^{2}+\frac{\zeta^{2} N^{2}}{2}\right) \frac{\partial}{\partial x}\left[\frac{1}{H(x)}\right]-z u w \frac{\partial^{2}}{\partial x^{2}}\left[\frac{1}{H(x)}\right]\right\}
$$

Integrating (6), the energy balance in the volume $V$ restricted by the free surface $z=0$, bottom $z=-H(x)$ and two arbitrary vertical lines $x=x_{1}$ and $x=x_{2}$ (see Fig. 1b) can be expressed as

$$
\frac{\partial}{\partial t} \int_{V} E d V+\int_{S} d \mathbf{S} \cdot \mathbf{F}=-\int_{V} R d V
$$

Here the divergence theorem $\int_{V} \boldsymbol{\nabla F} d V=\int_{S} d \mathbf{S} \cdot \mathbf{F}$ was used. The second term in (11) represents the energy flux through the contour $S$ (contour ABCD in Fig. 1b), which bounds the volume $V$. Obviously, the energy flux through the bottom (contour $\mathrm{CD}$ ) and free surface (contour $\mathrm{AB}$ ) equals zero if the "rigid lid" (at $z=0$ ) and "slip" [at $z=-H(x)$ ] conditions are used. The baroclinic energy flux $\mathbf{F}$ through the vertical sections $x=x_{1}$ and $x=x_{2}$ is nonzero in a very general case. However, if we consider the "localized" wave disturbance like internal solitary wave, and the boundaries $x_{1}$ and $x_{2}$ are placed quite far from the center of the soliton as presented in Fig. 1b, the integral $\int_{S} d \mathbf{S} \cdot \mathbf{F}$ is equal to zero because of the exponential decrease of all wave characteristics from the wave center.

Thus, the energy balance equation in (11) for ISWs over an inclined bottom [(4)] reads

$$
\frac{\partial}{\partial t} \int_{V} E d V=-\Psi_{0} \bar{\rho}_{0} \int_{V}\left\{\left(u^{2}-w^{2}+\frac{\zeta^{2} N^{2}}{2}\right) \frac{\partial}{\partial x}\left[\frac{1}{H(x)}\right]-z u w \frac{\partial^{2}}{\partial x^{2}}\left[\frac{1}{H(x)}\right]\right\} d V
$$

Substituting (4) into (12), the energy balance equation is simplified as follows:

$$
\frac{\partial}{\partial t} \int_{V} E d V=-\gamma \Psi_{0} \bar{\rho}_{0} \int_{V}\left(u^{2}-w^{2}+\frac{\zeta^{2} N^{2}}{2}-\gamma z u w\right) \frac{d V}{H(x)}
$$

Analysis of (13) shows that the growth or decrease of the wave energy (or wave amplitude) depends upon the sign of the two parameters, $\gamma$ and $\Psi_{0}$ : a positive or negative value of $\gamma$ shows the direction of wave propagation (upslope or downslope); the sign of the barotropic tidal flux, $\Psi_{0}$, denotes whether the waves move upstream or downstream. Combinations of these factors result in four different scenarios of wave evolution over the inclined bottom depending on the direction of wave propagation with respect to the bottom topography and the background current. They are summarized in Fig. 2 . It is clear from (13) that the energy of internal waves propagating upslope-upstream and downslopedownstream is amplified, whereas the waves propagating downslope-upstream or upslope-downstream lose their energy.

Further, we use (12) and (13) for quantitative estimations of the change of the energy of internal waves due to wave-flow interactions. Four scenarios of the evolution of energy of the wave depicted in 


\begin{tabular}{|c|c|c|}
\hline \multirow[b]{2}{*}{ Scenario } & $\begin{array}{c:c}\text { Downslope- } & \text { Upslope } \\
\text { upstream } & \text { upstream }\end{array}$ & $\begin{array}{l:c}\text { Downslope- } & \text { Upslope- } \\
\text { downstream } & \text { downstream }\end{array}$ \\
\hline & $\underset{\pi}{\rightarrow} \underset{\rightarrow}{\rightarrow}$ & 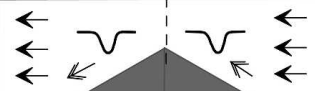 \\
\hline $\begin{array}{l}\text { Bottom } \\
\text { inclination }\end{array}$ & $\begin{array}{l:l}\gamma>0 & \gamma<0\end{array}$ & $\gamma<0$ \\
\hline $\begin{array}{l}\text { Direction } \\
\text { of flow }\end{array}$ & $\Psi_{0}>0$ & $\Psi_{0}<0$ \\
\hline Effect & Suppression A mplification & Amplification \\
\hline
\end{tabular}

FIG. 2. Four scenarios of internal wave propagation above the inclined bottom topography accompanied by the background barotropic current.

Fig. 2 are examined with the help of a numerical model.

\section{Scenarios of wave-flow interaction over an inclined bottom}

The wave-flow interaction is investigated numerically considering a typical oceanic situation of ISWs propagation in the slope-shelf area where bottom topography defined by (4) varies from 150 to $70 \mathrm{~m}$. In this series of numerical runs (the details of the model are described in the appendix with the fluid stratification presented by Fig. A1) we assume that a plane solitary internal wave of depression with amplitude $20 \mathrm{~m}$ or a packet of ISWs (with amplitudes 20, 17.5, 15, and 10 $\mathrm{m})$ propagates from the deep part of the basin to a shallow region (or from the shallow part to the deep). In addition to the waves, a barotropic current is also introduced into the system. To get quantitative estimates of how a strong stationary background current affects the waves propagating over an inclined bottom, four possible cases-(a) upslope-upstream propagation, (b) upslope-downstream propagation, (c) downslope-downstream propagation, (d) downslopeupstream propagation-are studied. The evolution of isopycnal $1022 \mathrm{~kg} \mathrm{~m}^{-3}$ is hereinafter used for the analysis of ISW evolution.

\section{a. Upslope-upstream propagation: Wave amplification}

Figure 3 shows the shoaling of four rank-ordered ISWs propagating upstream. In this experiment the value of the barotropic current was chosen in such a way to provide the critical condition for the first ISW at the depth of $90 \mathrm{~m}$ where the phase speed of the wave $c$

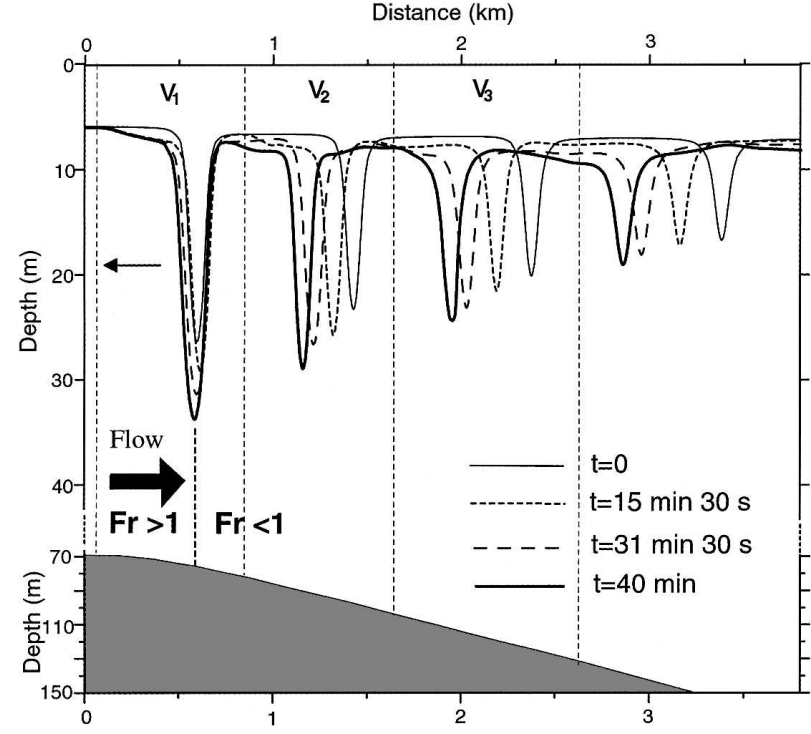

FIG. 3. The shoaling of four internal solitary waves propagating upstream. The $1022 \mathrm{~kg} \mathrm{~m}^{-3}$ isopycnal is shown by thin solid $(t=$ $0)$, dotted $(t=15 \mathrm{~min} 30 \mathrm{~s})$, dashed $(t=31 \mathrm{~min} 30 \mathrm{~s})$, and thick solid $(t=40 \mathrm{~min})$ lines. The position of the critical Froude number $\mathrm{Fr}=1$ is given by a vertical thick dashed line. All other parameters were $H_{0}=70 \mathrm{~m}, \gamma=-2.4 \times 10^{-4} \mathrm{~m}^{-1}$, and $\Psi_{0}=60 \mathrm{~m}^{2} \mathrm{~s}^{-1}$.

coincides with the velocity of flow $U$, and thus the Froude number

$$
\mathrm{Fr}=\frac{U}{c}
$$

is equal to unity. So, the flux is supercritical $(\mathrm{Fr}>1)$ for the waves to the left of the dashed vertical line and subcritical $(\mathrm{Fr}<1)$ to the right of it (Fig. 3). The evolution of the packet of ISWs is shown for 40-min time span.

As it is seen, the leading ISW is arrested by the downstream current (it does not propagate upslope). The amplitude of the wave gradually increases as the tidal flux supplies energy to the wave. A simple estimation shows that the permanent wave-flow feedback leads to the increase of the wave amplitude by almost $35 \%$ during $40 \mathrm{~min}$.

Three successive waves are not arrested by the incoming flux due to subcritical conditions $(\mathrm{Fr}<1)$ for them, but they are remarkably decelerated by current. The growth of their amplitudes is also evident in Fig. 3 by $31 \%, 27 \%$, and $22 \%$ for the second, third, and forth waves, respectively. Being smaller in amplitude these waves have smaller velocities $u$, which is why their growth is not so pronounced in comparison with the first wave [see (13)].

The growth of the energy of waves can be estimated 
from the energy balance equation in (12). The relative energy rate

$$
\alpha=\frac{\partial}{\partial t} \int_{V} E d V / \int_{V} E d V
$$

calculated at $t=0$ for the first three waves (thin solid line in Fig. 3), is equal to $\alpha_{1}=2.13 \times 10^{-4} \mathrm{~s}^{-1}, \alpha_{2}=$ $1.41 \times 10^{-4} \mathrm{~s}^{-1}$, and $\alpha_{3}=0.93 \times 10^{-4} \mathrm{~s}^{-1}$, for the first, second, and third waves, respectively. The integrals for the three waves in (15) were taken in volumes $V_{1}, V_{2}$, and $V_{3}$ schematically presented in Fig. 3. The horizontal interval for chosen volumes was taken in such a way to provide less than $0.5 \%$ of the maximum baroclinic signal at the lateral boundaries.

Note that for calculation of the Reynolds stresses (9), it was necessary to separate barotropic and baroclinic components of the wave field. Generally, the procedure of such a separation can be based on the independency of barotropic tidal flow on depth and the continuity equation. In our particular case the situation is even simpler because the exact analytical solution for barotropic flow was found [see (5)]. Thus the baroclinic signal is just a difference between numerical and analytical solutions.

Simple estimations of $\alpha_{j} \Delta t(j=1,2,3)$ show that the energy of first three waves may increased by $51 \%, 34 \%$, and $22 \%$ during the time interval $\Delta t=40 \mathrm{~min}$. These values should be considered as underestimated values due to the fact that the wave velocities $u$ and $w$ were also increased during the 40-min wave-flow interaction, whereas in our estimations we used the initial values of $u$ and $w$ at time $t=0$.

\section{b. Upslope-downstream propagation: Wave suppression}

Quite an opposite situation to those considered above takes place when the waves propagate upslope accompanied by the background current. It is expected from (13) that in this case the energy of the waves will attenuate in the process of the wave-flow interaction. The reason is that the term $R$ changes its sign to the positive in comparison with that in the previous scenario.

This qualitative reasoning is confirmed by the results of the numerical experiments. They were performed in such a way as to compare two wave trains without external forcing and exposed to the accompanying background current. Initial conditions for both experiments were the same as in the previous case a (Fig. 3) when four rank-ordered waves were located just over the slope at $t=0$. The only difference from the previous

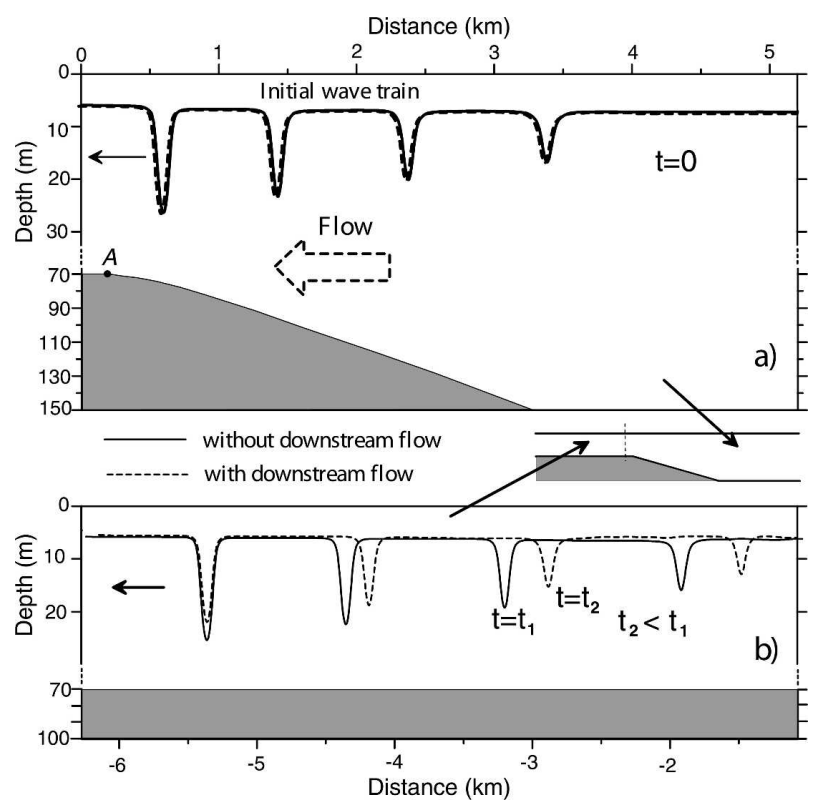

FIG. 4. Isopycnal $1022 \mathrm{~kg} \mathrm{~m}^{-3}$ shows four rank-ordered ISWs penetrating from the (a) deep $(150 \mathrm{~m})$ to the (b) shallow $(70 \mathrm{~m})$ water. The solid line represents waves in the absence of current $\left(\Psi_{0}=0\right)$; the waves moving with accompanying current $\left(\Psi_{0}=\right.$ $-60 \mathrm{~m}^{2} \mathrm{~s}^{-1}$ ) are shown by a dashed line. All other parameters were $H_{0}=70 \mathrm{~m}, \gamma=-2.4 \times 10^{-4} \mathrm{~m}^{-1}, t_{1}=147 \mathrm{~min}$, and $t_{2}=$ $65 \mathrm{~min}$.

case a is that the direction of barotropic flow was changed to the opposite one and this results in the acceleration of the propagating internal waves instead of their deceleration.

Isopycnal $1022 \mathrm{~kg} \mathrm{~m}^{-3}$ in Fig. 4 represents the profile of the wave train over the slope (Fig. 4a) and after its penetration into a shallow water (Fig. 4b). The solid line corresponds to the wave packet without the action of the background current, and the dashed line shows the same wave system after it was exposed to a strong accelerating current $\left(\Psi_{0}=-60 \mathrm{~m}^{2} \mathrm{~s}^{-1}\right)$.

Comparison of Figs. $4 \mathrm{a}$ and $4 \mathrm{~b}$ shows that both wave trains were "stretched" in the course of propagation. This effect takes place as a result of the nonlinear wave dispersion because waves with larger amplitudes propagate faster. For instance, the phase speeds of the first and fourth waves without barotropic current in the shallow water zone are 0.67 and $0.59 \mathrm{~m} \mathrm{~s}^{-1}$, respectively. The barotropic flow also plays an important role in the stretching of the wave packet. For the constant value of the flow discharge its speed $U$ increases from the tail of the packet to the leading wave because of the diminishing depth of water along the packet, which leads to the stretching of wave packet.

The next difference between scenarios $a$ and $b$ is that 


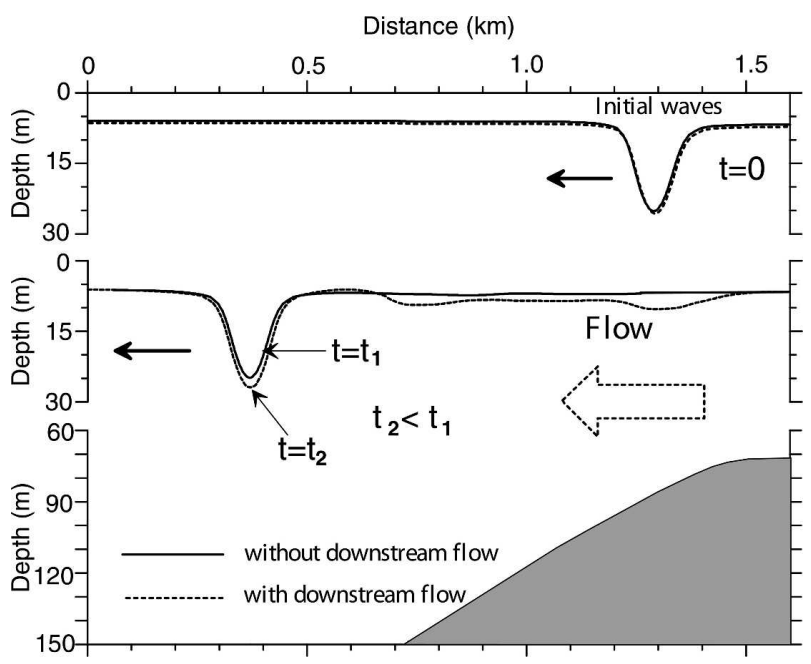

FIG. 5. Comparison of two waves propagating downstreamdownslope. The solid line shows the resulting wave in the absence of background flow, and the dashed line represents the wave profile after moving with the current $\left(\Psi_{0}=-60 \mathrm{~m}^{2} \mathrm{~s}^{-1}\right)$. Initial profiles at $t=0$ for both case are presented above. All other parameters were $H_{0}=70 \mathrm{~m}, \gamma=9.610^{-4} \mathrm{~m}^{-1}, t_{1}=20.5 \mathrm{~min}$, and $t_{2}=$ $12.5 \mathrm{~min}$.

the time of wave-flow interaction in the last case (the time of wave propagation over inclined bottom) is different for every particular wave. This time varies from $12.5 \mathrm{~min}$ for the leading wave which at $t=0$ is located near the shelf (the distance to point $\mathrm{A}$ is about $0.5 \mathrm{~km}$ in Fig. 4) to $87.5 \mathrm{~min}$ for the last wave, which at $t=0$ is $3 \mathrm{~km}$ away from point $\mathrm{A}$. As a consequence, the degree of damping for every particular wave in the train is different. The comparison of wave amplitudes in two wave trains (Fig. 4b) shows that in the course of waveflow interaction, the mean flow extracts energy from propagating waves and their amplitudes are decreased by $22 \%, 29 \%, 45 \%$, and $24 \%$ for the first, second, third, and fourth waves, respectively.

The last remark concerns the estimation of the wave energy changes due to wave-flow interaction, which can be done with the help of (12). The energy exchange rate $\alpha_{j}(j=1,2,3,4)$ in case $\mathrm{b}$ has the same value but its sign is opposite to the $\alpha_{j}$, which was estimated above for case a because of the different direction of the currents (initial conditions are identical in both cases).

\section{c. Downslope-downstream propagation: Wave amplification}

In this scenario the direction of propagation of the current and wave coincides; that is, both move downslope. Figure 5 represents two wave profiles: ISW

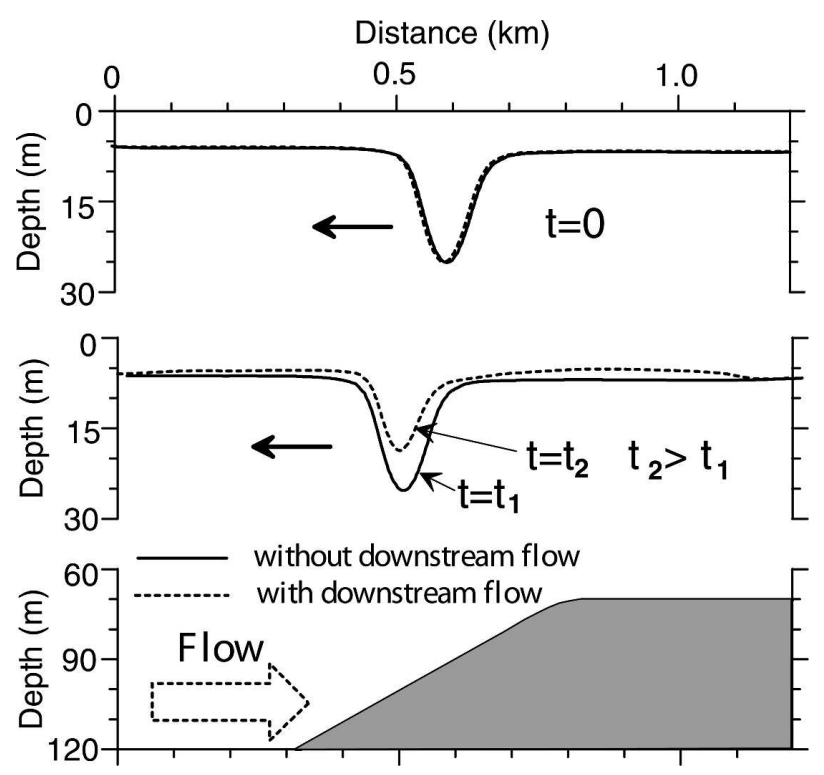

FIG. 6. The same as in Fig. 5 but for upstream wave propagation $\left(\Psi_{0}=60 \mathrm{~m}^{2} \mathrm{~s}^{-1}\right)$. All other parameters were $H_{0}=70 \mathrm{~m}, \gamma=9.6$ $10^{-4} \mathrm{~m}^{-1}, t_{1}=1 \mathrm{~min} 55 \mathrm{~s}$, and $t_{2}=12.5 \mathrm{~min}$.

propagating without tidal flux (solid lines) and under the action of the background current (dashed lines). The initial condition for both ISWs at $t=0$ was identical and both waves have amplitudes equal to $20 \mathrm{~m}$ (see upper panel in Fig. 5).

Taking into account that $\gamma>0$ and $\Psi_{0}<0$ in the present case, the right-hand side of (13) is positive. As a result, it is expected that the background current must amplify the propagating wave. Careful inspection of Fig. 5 confirms this conclusion. The comparison of the amplitudes of two waves moving with and without background current revealed a remarkable difference. The amplitude of the wave accelerating by the current is increased by $15 \%$ during the short time span $\Delta t=$ $12.5 \mathrm{~min}$ (cf. the wave profiles in Fig. 5). The energy exchange rate $\alpha$ calculated at $t=0$ is equal to $7.2 \times$ $10^{-4} \mathrm{~s}^{-1}$.

\section{d. Downslope-upstream propagation: Wave suppression}

The process of wave suppression of a single ISW with initial amplitude of $20 \mathrm{~m}$ propagating against the flow from the shallow water to the deep is shown in Fig. 6 by the dashed line. The phase speed of the ISW propagating upstream is a little larger than the velocity of the incoming flow in the present case. An averaged upstream wave velocity is $0.11 \mathrm{~m} \mathrm{~s}^{-1}$; that is, ISW is almost arrested by the flow. The amplitude of the wave, which is decelerated by the presence of the strong 
TABLE 1. Estimation of integrals defined by Eq. (16) for the four rank-ordered ISWs considered in scenarios (a) and (b).

\begin{tabular}{cccc}
\hline \hline Amplitude $(\mathrm{m})$ & $I_{2} / I_{1}$ & $I_{3} / I_{1}$ & $I_{4} / I_{1} \times 10^{3}$ \\
\hline 10 & 0.120 & 0.233 & 0.071 \\
15 & 0.089 & 0.176 & 0.053 \\
17.5 & 0.081 & 0.125 & 0.029 \\
20 & 0.073 & 0.106 & 0.021 \\
\hline
\end{tabular}

barotropic flux, rapidly decreases by $33 \%$ over 12.5 min. On the other hand, in the absence of the background flux the ISW propagates the same distance for only 1 min 55 s (solid line in Fig. 6).

Note that the change in the waveform is accompanied by changes in the background stratification. The stationary vertical flux over the inclined bottom presses the pycnocline against the free surface, which makes the ISW shorter.

\section{Discussion}

The energy balance equation developed in section 2 is formally valid for weak baroclinic disturbances, that is, when $\left|\mathbf{v}_{t}\right| \gg|\mathbf{v} \cdot \nabla \mathbf{v}|$ and $\left|\rho_{t}\right| \gg|\mathbf{v} \cdot \nabla \rho|$. Strong nonlinear internal waves that can be generated at the lee side of the obstacle are not included in this equation. Note, however, that the numerical results discussed in section 3 were obtained in the framework of a fully nonlinear model that incorporates all types of motions. The interpretation of the wave behavior (both for weak and strong waves) was given on the basis of the energy balance equation. So, it is possible to conclude that the developed theory can be used in a wider range than it can be formally applied to.

The calculations of the increment/decrement coefficient $\alpha$ in four scenarios have shown that not all terms in (12) or (13) equally contribute to the energy budget. This is clearly seen from Table 1 where the values of integrals

$$
\begin{aligned}
& I_{1}=\int_{V} u^{2} \frac{d V}{H(x)}, \quad I_{2}=\int_{V} w^{2} \frac{d V}{H(x)}, \\
& I_{3}=\int_{V} \frac{(\zeta N)^{2}}{2} \frac{d V}{H(x)}, \quad \text { and } I_{4}=\int_{V} \gamma z u w \frac{d V}{H(x)}
\end{aligned}
$$

are presented. Calculations were performed for the four rank-ordered ISWs considered in the first two scenarios (a) and (b) (see Figs. 3 and 4).

Scrutiny of Table 1 shows that the first term on the right-hand side of (13) plays a dominant role in the energy budget during the process of wave-flow inter- action. It provides more than two-thirds of the energy transfer from the barotropic flow to the internal waves. The next important factor in the attenuation or amplification of internal waves is the energy flux associated with the term $(\zeta N)^{2} / 2$ (potential energy). Table 1 shows that this flux comprises from $10 \%$ to $23 \%$ of the net energy exchange depending on wave amplitude. At the same time the term that is proportional to $w^{2}$ accounts for only $12 \%$ or less of the total energy flux. The last term in (13), which is proportional to $\gamma z u w$, gives infinitesimally small energy flux $I_{4}$ in comparison with $I_{1}$ and can be neglected. Thus, quantitative analysis of the energy balance equation in (12) shows that in many practical cases the rough estimations of energy balance can be simplified to the formula

$$
\frac{\partial}{\partial t} \int_{V} E d V \propto-\gamma \Psi_{0} \bar{\rho}_{0} \int_{V} \frac{u^{2}}{2 H(x)} d V .
$$

The considered mechanism of amplification and suppression of internal waves by barotropic flow over inclined bottom topography may have an important implication for the interpretation of tidally generated ISWs and wave trains in slope-shelf areas. The weakly nonlinear theory predicts the rank ordering of the waves in wave packets (Whitham 1974; Gerkema and Zimmerman 1995). Such a structure of internal wave trains is normally observed in the World Ocean (e.g., Osborn and Burch 1980; see also a review by Ostrovsky and Stepanyants 1989). However, some observations contradict the general conclusion derived from the weakly nonlinear theory. Several examples of such irregularity in the wave trains are discussed below.

A comprehensive study of large-amplitude internal solitary waves generated by tidal flow over the sharp Pearl Bank sill in the Sulu Sea (Apel et al. 1985) had shown that solitary waves were not rank ordered. The packet of ISWs becomes well formed with rankordered amplitude only after traveling a distance of approximately $200 \mathrm{~km}$ (Figs. 6 and 7 in Apel et al. 1985). The satellite data from the Defense Meteorological Satellite Program and Landsat imagery, which accompanied the measurements, had shown similar behavior within the wave packets.

Another experiment to measure tidally generated ISWs, the Synthetic Aperture Radar Internal Wave Signature Experiment (SARSEX), was conducted in the New York Bight (Liu 1988). Measurements at different moorings revealed that the waves were not rank ordered within the wave packets. For example the first four waves in the packet had amplitudes of 15, 4.5, 6.5, and $10 \mathrm{~m}$ at mooring 1 , and 11.6, 9.9,10.7, and $9.6 \mathrm{~m}$ at mooring 4 , respectively, which was deployed $14 \mathrm{~km}$ 
away from mooring 1 along the trace of the packet propagation (Fig. 13 in Liu 1988).

More recent observations of internal solitary waves during the Coastal Ocean Probing Experiment (COPE) maintain the idea that internal waves within packets very often have an irregular distribution of amplitude. The COPE took place at the east Pacific shelf in September 1995 (Kropfli et al. 1999; Stanton and Ostrovsky 1998). In particular, the thermistor chain data and radar signatures revealed that a strong internal wave front propagated to the shelf after being forced by a strong spring tide on 25 September 1995. The initial displacement was followed by a train of pulses; at least 10 of them had comparable amplitudes and were not rank ordered (Fig. 3 in Kropfli et al. 1999).

Knight Inlet, Canadian fjord (British Columbia), has recently become a very popular location for the experimental study of tidally generated internal waves. Highresolution measurements of the internal waves were performed by Farmer and Armi (1999a,b, 2002) with the use of various techniques. Figure 1 of Farmer and Armi (1999b) clearly shows the generation of firstmode nonlinear non-rank-ordered wave trains upstream of the sill. These waves were also detected recently by Cummins et al. (2003) with the use of an echo sounder. The irregular amplitude distributions of the waves across the sill are shown in Fig. 6 of the same paper.

To summarize, we can assume that one of the reasons of the irregularity of the observed internal waves in the packets could be the mechanism of suppression and amplification by a stratified tidal current over an inclined bottom topography described above. To prove this idea we present here an analysis of the numerical experiments performed in the Knight Inlet sill as a case study.

Case study: Modeling of stratified flow over the Knight Inlet sill

The Knight Inlet is a deep, long, and relatively narrow (average width of $2.9 \mathrm{~km}$ ) stratified fjord with a sharp sill that divides it into inner and outer basins. The semidiurnal tidal flow over the sill becomes supercritical throughout the part of the tidal cycle. This becomes clear by comparing the barotropic tidal velocity with the local phase speed of the first baroclinic mode $c$, which has a value in the range $0.5-0.55 \mathrm{~m} \mathrm{~s}^{-1}$ in summer stratification for a sill depth of 60 (seaward end) to 70 $\mathrm{m}$ (landward end). For the maximum value of the tidal velocity $U_{\max }$ being in the range $0.6-1.0 \mathrm{~m} \mathrm{~s}^{-1}$, this means that the flow becomes supercritical $(\mathrm{Fr}>1)$ in the sill-top area over majority of the tidal cycle.

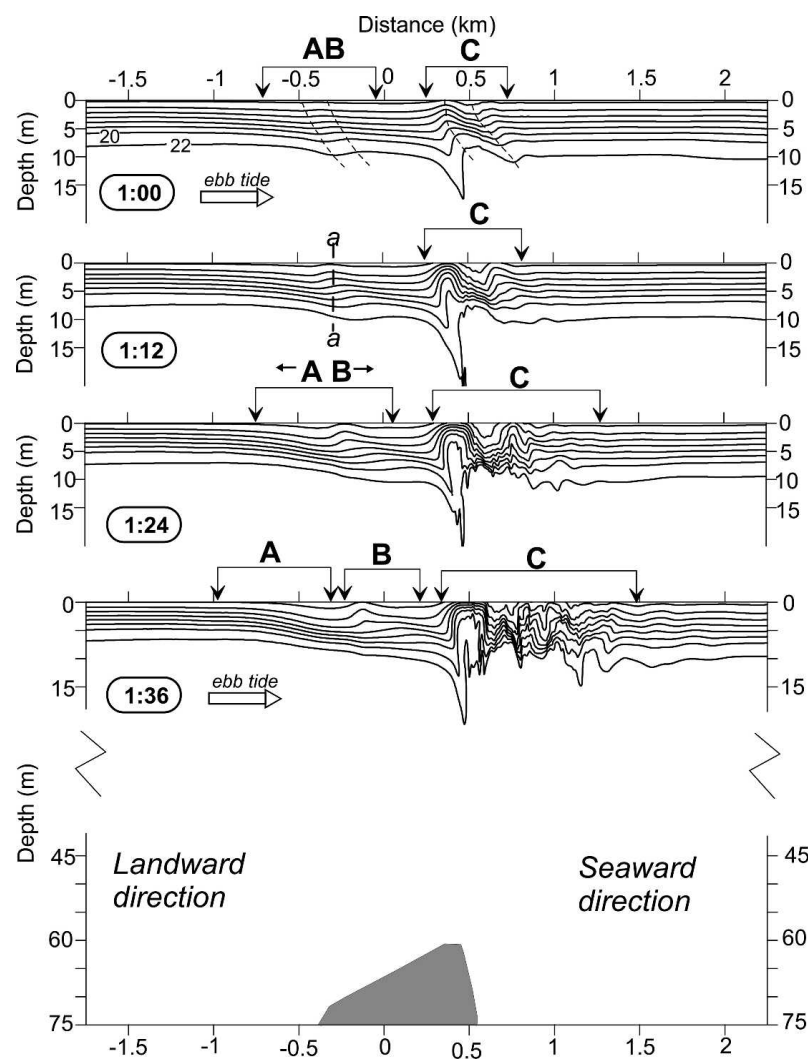

FIG. 7. The conventional density $\sigma_{t}\left(\mathrm{~kg} \mathrm{~m}^{-3}\right)$ obtained at the beginning of the ebb tide. The lines of equal phase for the wave fragments $\mathrm{AB}$ and $\mathrm{C}$ are shown by dashed lines. The multimodal structure is shown by the vertical section " $a-a$ ". The density contour interval is $2 \mathrm{~kg} \mathrm{~m}^{-3}$. The landward and seaward parts of the sill are shown.

Taking into account that the internal wave fronts are almost straight lines near the channel center (Farmer and Armi 1999b) the analysis of the generated waves was performed in the framework of a two-dimensional nonlinear nonhydrostatic numerical model, which reproduced all stages of topographic generation of internal waves (the details of the model are described in the appendix). The discharge of tidal flow was specified to be a harmonic function with period $T=12 \mathrm{~h}$ and with maximum velocity $U_{\max }=1.0 \mathrm{~m} \mathrm{~s}^{-1}$ above the top of the Knight Inlet sill.

Detailed numerical modeling of the stratified flow over the Knight Inlet sill was performed recently by Cummins et al. (2003). We concentrate here upon the irregular structure and dynamics of ISWs that were not described in the aforementioned paper.

Figure 7 represents the baroclinic response of the system to the external tidal forcing obtained for the summer stratification. The velocity of tide $U$ reaches the value of $0.5 \mathrm{~m} \mathrm{~s}^{-1}$ at time moment $t=1 \mathrm{~h}$ and the tidal flow above the top of the sill becomes critical 
$(\mathrm{Fr}=1)$ for internal waves. The formation of two systems of waves above the landward slope of the sill, fragment $\mathrm{AB}$, and behind its seaward slope, fragment $\mathrm{C}$, is clearly seen in Fig. 7. They evolve permanently as the tidal flow accelerates during the next $0.5 \mathrm{~h}$ (from $t=1 \mathrm{~h}$ to $t=1.36 \mathrm{~h}, \mathrm{Fr}>1$ ). In fact, these waves can be considered as a superposition of several baroclinic modes propagating upstream. The counterphase vertical displacement of isopycnals shown by the section " $a-a$ " at $t=1.12 \mathrm{~h}$ illustrates their multimodal structure. Because the phase speed of different modes depends upon the mode number, these waves begin to separate in space from each other as the tidal flux accelerates over the top of the sill. The second and the higher baroclinic modes are substantially slower than the tidal current. Therefore they are trapped by the tide and propagate downstream (seaward), whereas the wave of the first mode remains at the place of wave generation. It is evident that the wave A represents the first-mode baroclinic bore, whereas the system B possesses the characteristics of the second and higher baroclinic modes.

The further evolution of the waves A and B during the next time span (from $t=2 \mathrm{~h} 00 \mathrm{~min}$ to $2 \mathrm{~h} 24 \mathrm{~min}$ ) is given in Fig. 8. Here the density field along with the velocity and Richardson number fields $\left[\mathrm{Ri}=N^{2}(z) / u_{z}^{2}\right]$ are shown. The direction and value of the tidal velocity (a superposition of barotropic and baroclinic components) is indicated by the arrows. Zones of instability where $\mathrm{Ri}<1 / 4$ are shaded gray. They mostly occupy the bottom boundary layer at the top of the sill and its seaward flank. In this region the hydraulic jump is accompanied by well-developed water mixing.

Let us analyze the evolution of the wave fragment A. In fact this fragment is the first-mode baroclinic bore propagating upstream. In conjunction with opposing background tidal flux both create the specific structure of the velocity field, which is characterized by small values of currents above the pycnocline and strong currents below it. A similar distribution of tidal velocity above the sill was observed in the measurements (see, e.g., Figs. 7 and 8 in Farmer and Armi 1999a).

The propagating bore A disintegrates into a series of nonlinear internal waves during the time span from $t=$ $2 \mathrm{~h} 00 \mathrm{~min}$ to $t=2 \mathrm{~h} 24 \mathrm{~min}$. This process is seen in Figs. $8 \mathrm{~b}$ and $8 \mathrm{c}$ : the nonlinear dispersion of the bore transforms the trailing edge of the wave depression into short-period oscillations.

Note that the process of the nonlinear transformation of the baroclinic bore into a packet of nonlinear short waves in the present case is unique and distinctive from the usual one due to the critical condition, which occurs over the sill. The upstream propagating wave packet is

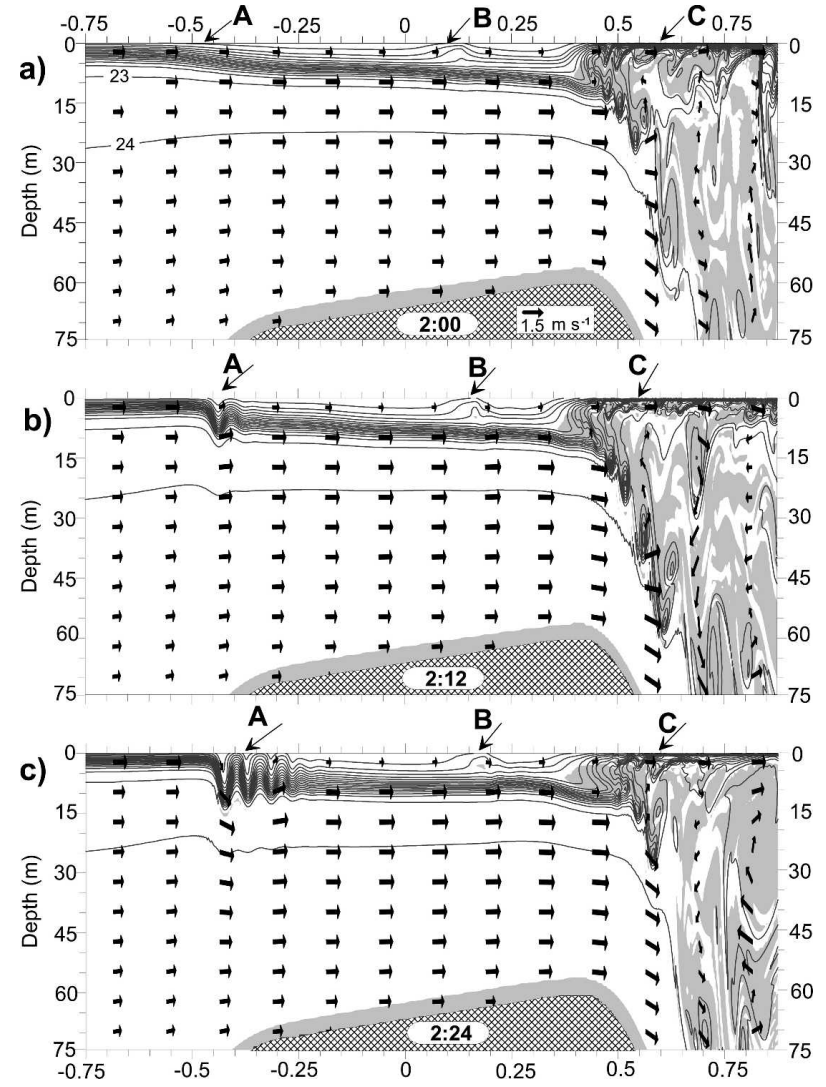

FIG. 8. The evolution of the conventional density $\sigma_{t}\left(\mathrm{~kg} \mathrm{~m}^{-3}\right)$ (isolines are shown by solid lines), velocity (the magnitude and the direction are given by arrows), and Richardson number fields (areas with $\mathrm{Ri}<1 / 4$ are shown and shaded in light gray) during the ebb tide for (a) $2 \mathrm{~h}$; (b) $2 \mathrm{~h} 12 \mathrm{~min}$; and (c) $2 \mathrm{~h} 24 \mathrm{~min}$. Letters $\mathrm{A}, \mathrm{B}$, and $\mathrm{C}$ show the wave fragments in Fig. 7. The density contour interval is $1 \mathrm{~kg} \mathrm{~m}^{-3}$.

arrested by the strong incoming tidal current just in the place where sharp changes of the bottom topography take place. As a result the internal waves within the wave train are located at different depths $H(x)$ (see Fig. 8c).

This, in turn, leads to the situation where the mechanism of wave-flow interaction discussed in sections 2 and 3 can lead to the irregular structure of the wave packet. The analysis of subsequent stages of the evolution of the wave train confirms this assumption. Figure 9 shows the behavior of the wave packet A by isopycnal $1022 \mathrm{~kg} \mathrm{~m}^{-3}$ between $2 \mathrm{~h} 36$ min and $3 \mathrm{~h} 00$ min with a 3 -min time step. The amplitudes of the waves are changed chaotically.

Figure 8 shows that at $t=2 \mathrm{~h} 24 \mathrm{~min}$ the wave packet has a regular structure: wave amplitudes decrease gradually from the leading wave to the wave tail. However, by the time moment $t=2 \mathrm{~h} 48$ min the amplitudes of first five waves become comparable in Fig. 9. During 

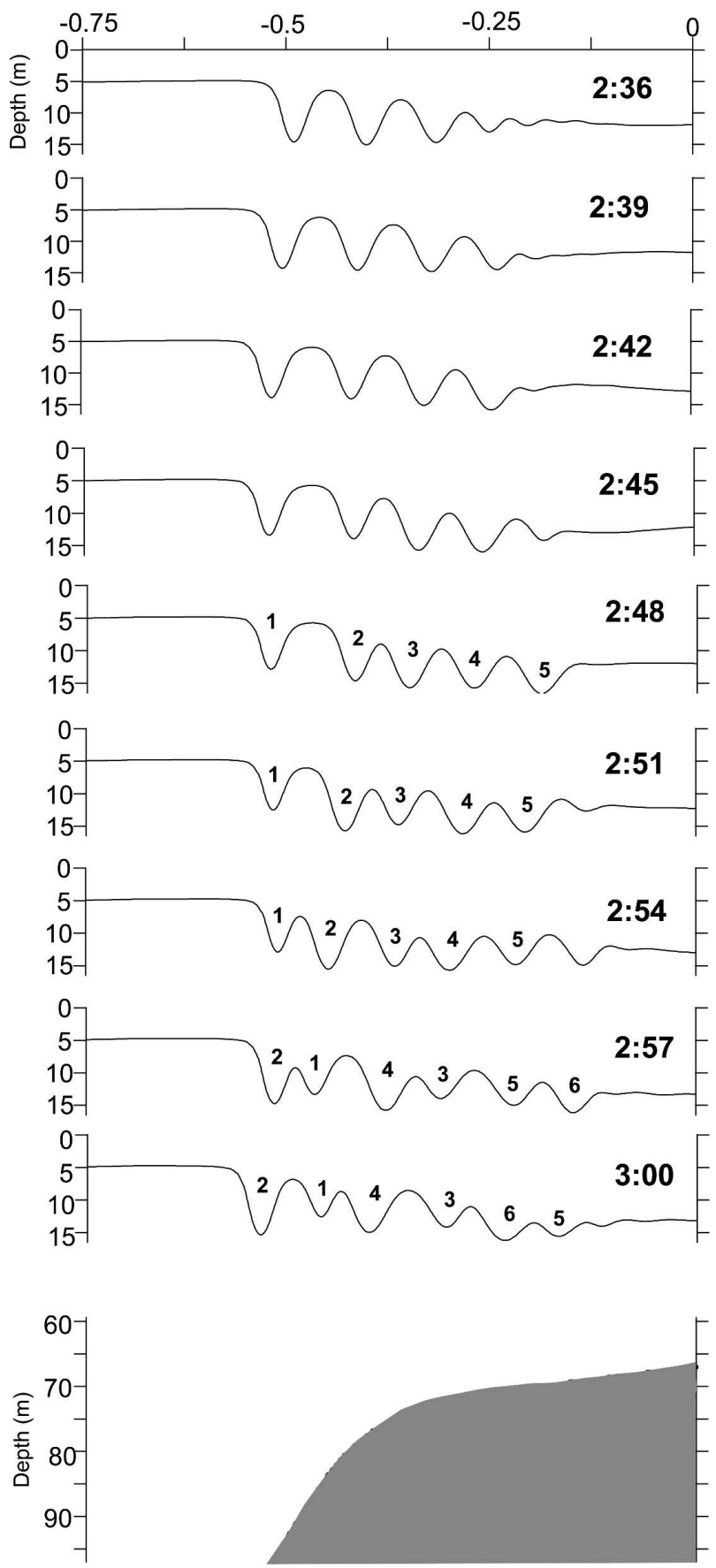

FIG. 9. Evolution of the $1022 \mathrm{~kg} \mathrm{~m}^{-3}$ isopycnal over the Knight Inlet sill for the time span from $2 \mathrm{~h} 36 \mathrm{~min}$ to $3 \mathrm{~h}$. Waves are marked by numbers $1,2,3,4,5$, and 6 .

the time span $t=2 \mathrm{~h} 48$ min $-3 \mathrm{~h} 00$ min the wave amplitudes start to change chaotically: the amplitudes of the second and the fourth waves become larger, whereas the amplitudes of the first and third waves are substantially smaller.

The reason for this "leapfrog" behavior of the waves becomes understandable if one assumes that two different processes act together: (i) the exchange of energy between waves and flow and (ii) the interaction of solitary waves.

\section{1) ENERgy EXCHANGe BETWEen WAVES AND FLOW}

While the wave packet is arrested by the incoming tidal flux over the sill, the water depth in the place of every individual wave in the packet is different: it is $90 \mathrm{~m}$ for the leading wave at $t=2 \mathrm{~h} 36 \mathrm{~min}$ and decreases to $70 \mathrm{~m}$ in the wave tail (see Fig. 9). Taking into account that the value of tidal velocity depends on the water depth $H(x)$, namely, $U(x, t)=\Psi_{0} / H(x) \sin (\omega t)$, the value of $U(x, t)$ increases from the head to the tail of the wave packet and the leading and subsequent waves have different background conditions with respect to the depth and tidal velocity.

If the energy of the wave (or, alternatively, wave amplitude) is assumed to be changed due to the waveflow interaction, then Fig. 9 shows that the energy exchange between tidal current and waves is more favorable for the wave tail where tidal flow is stronger, than for the leading wave, where the depth of the basin is deeper. Evidence of such suppression is illustrated by Fig. 9 for time span $2 \mathrm{~h} 51 \mathrm{~min}$. This is in accordance with (12) and (13), which show that the basin depth is present in the denominator.

On the other hand, from (12) and (13) it is also clear that the energy flux depends upon the steepness and curvature of the bottom [in other words upon the first and second derivatives of $H(x)]$. The flux is larger for more steep and more curved topographies in places where the vertical velocity $W$ and its horizontal derivative $W_{x}$ are larger. In this context the landward flank of the Knight Inlet sill is a more preferable place for wave-flow interaction. These two contradictive tendencies may make an analysis of any concrete real situation quite cumbersome.

\section{2) INTERACTION BETWEen SOLITARy WAVES}

The second mechanism, namely, wave-wave interaction, must also be taken into account for the explanation of the wave evolution at time span 2 h 54 min$3 \mathrm{~h} 00 \mathrm{~min}$, when the leading wave (now it is wave 2 instead of wave 1) again becomes larger. A similar effect takes place with the third and the fourth waves. Bearing in mind that the phase speed of nonlinear waves depends on their amplitude (largest wave propagates faster) waves 2 and 4 must overtake waves 1 and 3 , respectively. In other words, the collision of two pairs of ISWs, namely, 1-2 and 3-4, takes place. The change 


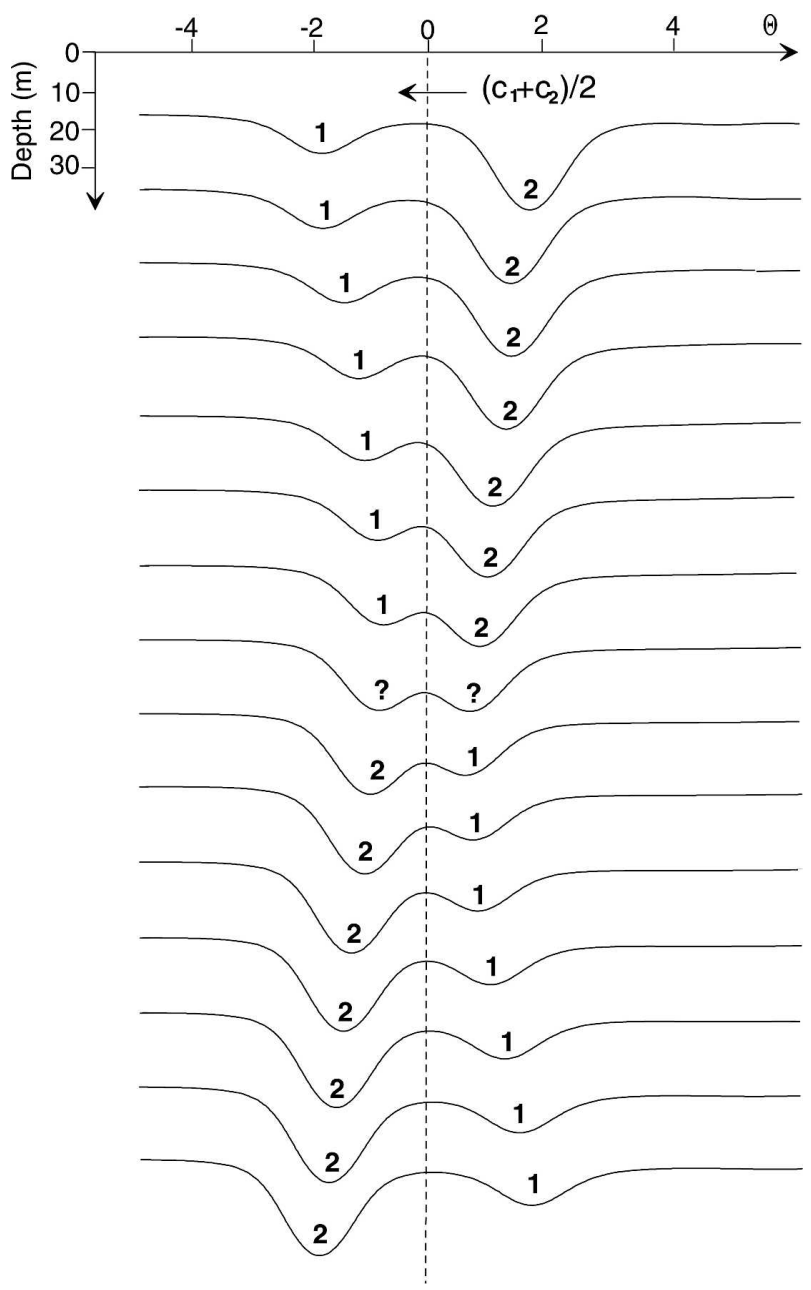

FIG. 10. Model-predicted collision of two strong internal solitary waves in the coordinate system moving with velocity $\left(c_{1}+\right.$ $\left.c_{2}\right) / 2$. Here $\Theta=\left[x-t\left(c_{1}+c_{2}\right)\right] /\left(\lambda_{1}+\lambda_{2}\right) / 2 ; c_{1}, \lambda_{1}$ and $c_{2}, \lambda_{2}$ are the phase speed and wavelength of the first and second waves, respectively. The time step between plots is equal to $125 \mathrm{~s}$.

of the wave amplitude and order in the wave packet over the time span 2 h 51 min- 3 h 00 min can be interpreted in terms of this mechanism: waves 2 and 4 gradually transform their energy to waves 1 and 3 , respectively, and by the time $t=3 \mathrm{~h}$ this process is completed (waves exchange their positions).

To illustrate the latter process more clearly (collision of two ISWs) Fig. 10 shows "pure" wave-wave interaction without tidal current. Here two ISWs with amplitudes 10 and $20 \mathrm{~m}$ propagating with phase speed $c_{1}$ and $c_{2}\left(c_{2}>c_{1}\right)$ in the same direction over a constant depth $(H=70 \mathrm{~m})$ are shown [the coordinate system is moving with velocity $\left.\left(c_{1}+c_{2}\right) / 2\right]$. The amplitudes of waves 1 and 2 are remarkably different. Wave 2, being larger and faster, overtakes and suppresses wave 1 . In fact, the second wave transfers its energy during the nonlinear collision with the first wave and the first wave runs away when this process is completed. This numerical experiment was performed in the framework of the model described in section a of the appendix.

\section{Summary and conclusions}

The problem of energy exchange between the internal waves and barotropic current over inclined bottom topography is studied theoretically and in the framework of a fully nonlinear nonhydrostatic numerical model. The analytical investigation performed with the use of the energy balance equation derived for the continuously stratified fluid and the results of numerical runs show that energy can transfer either toward or from the internal wave depending on the direction of propagation of the wave with respect to the current and bottom topography. Four scenarios of wave-flow interaction over the inclined bottom were considered: upslope-upstream, downslope-downstream, downslopeupstream, and upslope-downstream. In the first two cases there is transfer of energy from the background tidal flow to the propagating waves, which grow in amplitude due to the wave-flow interaction. In two last cases, the wave loses its energy (transfers to the current) and its amplitude reduces.

As distinct from many traditional studies of the problem of wave-flow interaction when vertical shear of the background current $U_{z}$ is considered (see, e.g., Baines 1995) as a basic reason and source of energy exchange, an important implication from the present work is that the rate of amplification or suppression of internal waves over an inclined bottom depends basically upon the horizontal shear of the tidal current $U_{x}$ and $W_{x}$. This fact is seen from (6) and (9). Derivative $U_{z}$ can be omitted from the analysis as a second-order effect because the tidal current is basically homogeneous in the vertical direction-see (5) (if the influence of thin bottom boundary layer is not considered).

The horizontal shear $U_{x}$ and $W_{x}$ in turn depends upon the characteristics of the bottom topography: local depth $H(x)$, bottom inclination, $\partial H(x) / \partial x$, and bottom curvature, $\partial^{2} H(x) / \partial x^{2}$. The results of the numerical runs on the propagation of solitary internal waves over an inclined bottom on the background of accelerating or decelerating barotropic flow confirm this conclusion derived from the energy balance equation.

These results on the possible amplification or suppression of the internal waves during wave-flow interaction over inclined bottom may have an important implication for the interpretation of oceanographic data on baroclinic tides observed in many slope-shelf areas. Particularly, this mechanism gives a reasonable explanation of the nature of the non-rank-ordered structure 
of tidally generated packets of internal waves often observed in many sites of the World Ocean.

Acknowledgments. This work was supported by the U.K. Natural Environment Research Council Grant NE/C50747X/1 on the dynamics of jet-type fjord. We thank Nigel Aird for his helpful discussions. We thank the anonymous reviewers, whose comments have led to significant improvement of the paper.

\section{APPENDIX}

\section{The Numerical Model}

In terms of the streamfunction $\psi$ and the vorticity $\Omega$, $u=\psi_{z}, \quad w=-\psi_{x}, \quad$ and $\quad \Omega=\psi_{x x}+\psi_{z z}$,

the Reynolds system of equations in the Boussinesq approximation reads (Vlasenko et al. 2005b)

$$
\begin{aligned}
\Omega_{t}+J(\Omega, \psi) & =g \rho_{x} / \bar{\rho}_{0}+A_{H} \Omega_{x x}+\left(A_{V} \psi_{z z}\right)_{z z}+\left(A_{V} \psi_{x z}\right)_{x z} \quad \text { and } \\
\rho_{t}+J(\rho, \psi)+\bar{\rho}_{0} / g N^{2}(z) \psi_{x} & =K_{H} \rho_{x x}+\left[K_{V}(\bar{\rho}+\rho)_{z}\right]_{z} .
\end{aligned}
$$

Here $A_{V}, K_{V}, K_{H}$, and $A_{H}$ are the coefficients of vertical and horizontal eddy viscosity and turbulent diffusion, $J(A, B)=A_{x} B_{z}-A_{z} B_{x}$ is the Jacobian operator, and $\bar{\rho}=\bar{\rho}(z)$ and $\rho=\rho(x, z, t)$ are the background and wave perturbation densities.

The "rigid lid" boundary condition

$$
\psi(x, 0, t)=G, \quad \Omega=0, \quad \text { and } \rho_{z}=0
$$

is used at the free surface. Here $G$ is equal to either $\Psi_{0}$ when the problem of wave-flow interaction is considered, or $\Psi_{0} \sin (\omega t)$ for the problem of generation of baroclinic tides ( $\Psi_{0}$ is the water discharge through the vertical cross section).

The contour $z=-H(x)$ is a streamline through which zero mass flux is required:

$$
\psi=0, \quad \Omega=\Omega_{0}, \quad \text { and } \quad \rho_{\mathbf{n}}=0,
$$

where $\mathbf{n}$ is a vector normal to the bottom. The vorticity $\Omega_{0}$ at the bottom is calculated from the streamfunction at the previous temporal step.

At the lateral "liquid" boundaries $x= \pm l$ of the calculation domain, "zero" values of baroclinic perturbation are required. To avoid the influence of reflection from the lateral boundaries on the model results, the boundaries were distanced sufficiently far from the topography so that the leading waves propagating from the source of generation do not reach the boundaries during the numerical experiment.

System (A2)-(A4) along with the appropriate initial conditions for all variables ("zero" conditions for the problem of baroclinic tides generation or initial "wave" conditions for the problem of wave evolution) was solved numerically by means of the alternating direction implicit method. More details on the numerical procedure are presented in Vlasenko et al. (2005b).

\section{a. Propagation of IWS}

In the problem wave-flow interaction (section 3 ) zero coefficients $A_{V}$ and $K_{V}$ were used, and $A_{H}$ and $K_{H}$ were equal to $10^{-4} \mathrm{~m}^{2} \mathrm{~s}^{-1}$. These values were taken as small as possible to minimize the influence of the viscosity on the model results on the one hand, and at the same time to secure the numerical stability of the scheme on the other hand.

The model was initialized in a way similar to that of Vlasenko and Hutter (2002). To obtain the initial fields for the incident wave, a basin with constant $150-\mathrm{m}$ depth was considered at the initial stage. The corresponding density profile is shown in Fig. A1 (Vlasenko et al. 2005a). For initialization we used the first-mode analytical solitary wave solution of the Korteweg-de Vries equation. Such an initial field represents a stationary solitary wave in a weakly nonlinear case, which does not satisfy the system (A2) for a strong ISW. Being inserted in the numerical scheme, the largeamplitude ISW will evolve until a new stationary solitary wave is formed at the frontal side of the wave field and the leading wave separates from the dispersive wave tail. This leading ISW is then used as an initial condition for the problem of the interaction of intense ISWs with the bottom topography. The stationary background current is introduced into the model at the next stage as a key factor to study the wave-flow interaction over an inclined bottom.

\section{b. Generation of ISWs by interaction of the tide with the Knight Inlet sill}

An oscillating tidal flow $G=\Psi_{0} \sin (\omega t)$ where $\omega$ is the semidiurnal tidal frequency is used as an external forcing in (A3). The vertical turbulent viscosity $A_{V}$ and diffusivity $K_{V}$ are determined by the Richardson number-dependent parameterizations (Pacanowski and Philander 1981), namely,

$$
A_{V}=\frac{A_{0}}{(1+\beta \mathrm{Ri})^{k}}+A_{b} \quad \text { and } \quad K_{V}=\frac{A_{V}}{1+\beta \mathrm{Ri}}+K_{b} .
$$

Here $A_{b}$ and $K_{b}$ are the background dissipation parameters, and $A_{0}, \beta$, and $k$ are the adjustable parameters. 


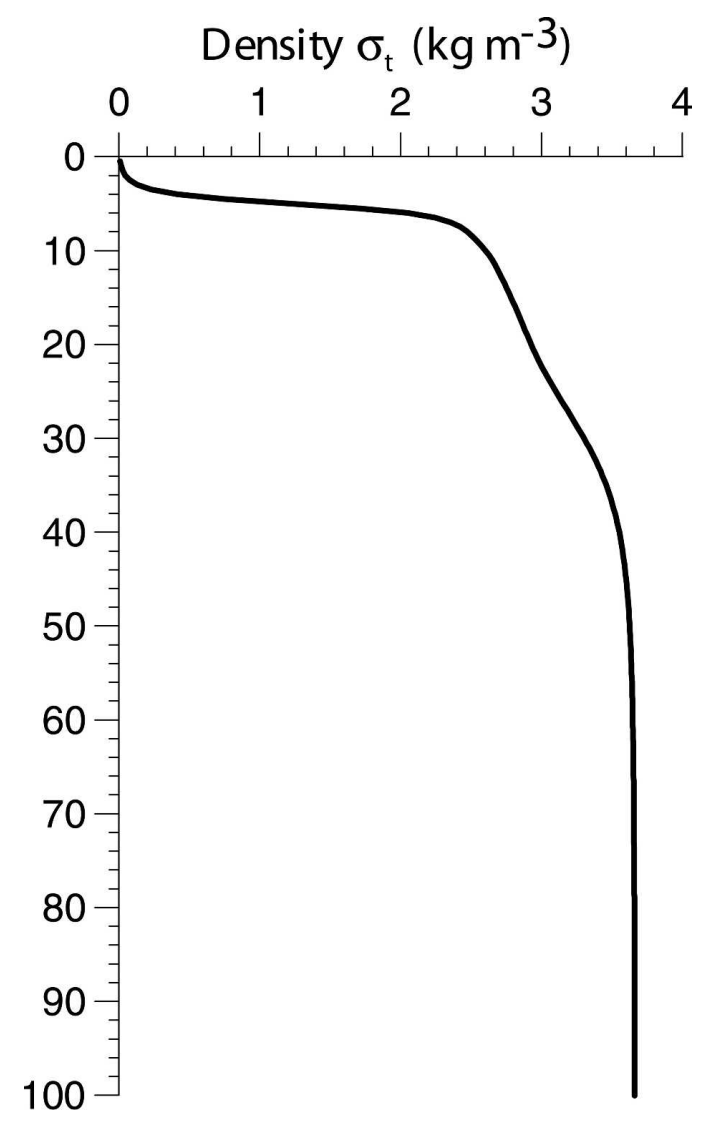

FIG. A1. Density profile used in section 3.

Several numerical experiments were performed to determine the values of the eddy viscosity and turbulent diffusivity parameters $A_{0}, A_{b}, k, \beta$, and $K_{b}$. It was found that by taking $\Delta t=1 \mathrm{~s}, \Delta x=2.5 \mathrm{~m}, \Delta z=0.3-1.0 \mathrm{~m}$ (to the left of the sill), $A_{0}=10^{-3} \mathrm{~m}^{2} \mathrm{~s}^{-1}, A_{b}=10^{-3} \mathrm{~m}^{2} \mathrm{~s}^{-1}$, $k=1, \beta=1$, and $K_{b}=10^{-4} \mathrm{~m}^{2} \mathrm{~s}^{-1}$, the numerical scheme was quite stable. The coefficients of horizontal turbulent viscosity $A_{H}$ and diffusivity $K_{H}$ were equal to $0.1 \mathrm{~m}^{2} \mathrm{~s}^{-1}$.

A typical summer distribution of density (Fig. A2a) is used in the model calculations where the upper thin $(<10 \mathrm{~m})$ layer comprises nearly freshet $\left(\sigma_{t}=8-10 \mathrm{~kg}\right.$ $\mathrm{m}^{-3}$ ) in contrast to bottom waters, which are weakly stratified with $\sigma_{t}=24-25 \mathrm{~kg} \mathrm{~m}^{-3}$.

The bottom profile of the sill with its minimum depth of $60 \mathrm{~m}$ is schematically shown in Fig. A2b. The model topography of the tested area includes a realistic representation of the Knight Inlet sill over $4 \mathrm{~km}$ of the calculational domain. For simplicity, the depth beyond the area of the sill is set to be constant: $150 \mathrm{~m}$ seaward and $200 \mathrm{~m}$ landward. The shape of the sill is asymmetric: the seaward slope is steeper than its landward counterpart.

The next important input parameter is the value of the tidal discharge $\Psi_{0}=U(x) H(x)=U_{\max } H_{\text {top }}$, where $U_{\max }$ is the maximum value of the barotropic tidal velocity above the highest point of the sill (see Fig. 2b). The baroclinic tidal response occurring in the sill area strongly depends upon the strength of the tidal current. The value $U_{\mathrm{max}}$, estimated by Farmer and Smith (1980), varies in the range $0.6-1.0 \mathrm{~m} \mathrm{~s}^{-1}$. The careful measurements performed by Klymak and Gregg (2001) in the sill area revealed the maximum flow velocity to be approximately $1.2 \mathrm{~m} \mathrm{~s}^{-1}$.

\section{REFERENCES}

Apel, J. R., J. R. Holbrook, A. K. Liu, and J. J. Tsai, 1985: The Sulu Sea internal soliton experiment. J. Phys. Oceanogr., 15, $1625-1661$.

\section{Density $\sigma_{\mathrm{t}}\left(\mathrm{kg} \mathrm{m}^{-3}\right)$}
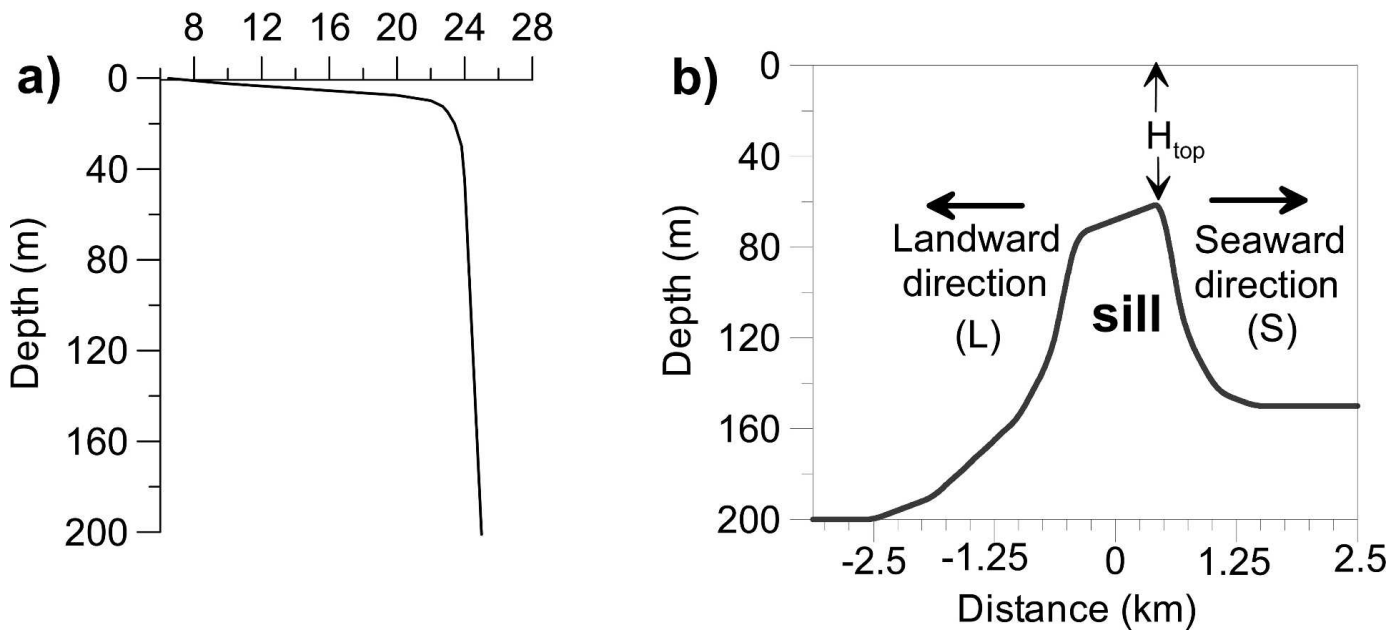

FIG. A2. (a) Typical density profile for summer stratification in Knight Inlet sill area. (b) Bottom topography in the sill area. 
Baines, P. G., 1995: Topographic Effects in Stratified Flows. Cambridge University Press, 482 pp.

Clarke, S. R., and R. H. Grimshaw, 1999: The effect of weak shear on finite-amplitude internal solitary waves. J. Fluid Mech., 395, 125-159.

Cummins, P. F., and M. Li, 1998: Comment on "Energetics of borelike internal waves" by Frank S. Henyey and Antje Hoering. J. Geophys. Res., 103, 3339-3341.

— S. Vagle, L. Armi, and D. M. Farmer, 2003: Stratified flow over topography: Upstream influence and generation of nonlinear internal waves. Proc. Roy. Soc. London, 459, 14671487.

Djordjevic, V. D., and L. G. Redekopp, 1978: The fission and disintegration of internal solitary waves moving over twodimensional topography. J. Phys. Oceanogr., 8, 1016-1024.

Farmer, D., and D. Smith, 1980: Tidal interaction of stratified flow with a sill in Knight inlet. Deep-Sea Res., 27A, 239-254.

_ , and L. Armi, 1999a: Stratified flow over topography: The role of small-scale entrainment and mixing in flow establishment. Proc. Roy. Soc. London, 455, 3221-3258.

— waves over topography. Science, $\mathbf{2 8 3}, \mathbf{1 8 8 - 1 9 0 .}$

— fronts and transition to the uncontrolled state. Proc. Roy. Soc. London, 458, 513-538.

Gerkema, T., and J. T. F. Zimmerman, 1995: Generation of nonlinear internal tides and solitary waves. J. Phys. Oceanogr., 25, 1081-1094.

Henyey, F. S., and A. Hoering, 1997: Energetics of bore-like internal waves. J. Geophys. Res., 102, 3323-3330.

Horn, D. A., L. G. Redekopp, J. Imberger, and G. N. Ivey, 2000: Internal wave evolution in a space-time varying field. J. Fluid Mech., 424, 279-301.

Klymak, J. M., and M. C. Gregg, 2001: Three-dimensional nature of flow near a sill. J. Geophys. Res., 106, 22 295-22 311.

Kropfli, R. A., L. A. Ostrovski, T. P. Stanton, E. A. Skirta, A. N. Keane, and V. Irisov, 1999: Relationships between strong internal waves in the coastal zone and their radar and radiometric signatures. J. Geophys. Res., 104, 3133-3148.
LeBlond, P. H., and L. A. Mysak, 1979: Waves in the Ocean. Elsevier, 602 pp.

Lee, C.-Y., and R. C. Beardsley, 1974: The generation of long nonlinear internal waves in a weakly stratified shear flow. $J$. Geophys. Res., 79, 453-462.

Liu, A. K., 1988: Analysis of nonlinear internal waves in the New York Bight. J. Geophys. Res., 93, 12 317-12 329.

Long, R. R., 1970: Blocking effects in flow over obstacles. Tellus, 22, 471-480.

Maslowe, S. A., and L. G. Redekopp, 1980: Long nonlinear waves in stratified shear flows. J. Fluid Mech., 101, 321-348.

Osborn, A. R., and T. L. Burch, 1980: Internal solitons in the Andaman Sea. Science, 208, 451-460.

Ostrovsky, L. A., and Y. A. Stepanyants, 1989: Do internal solitons exist in the ocean? Rev. Geophys., 27, 293-310.

Pacanowski, R. C., and S. G. H. Philander, 1981: Parameterization of vertical mixing in numerical models of tropical oceans. $J$. Phys. Oceanogr., 11, 1443-1451.

Pelinovsky, E. N., Y. A. Stepanyants, and T. G. Talipova, 1995: Simulation of nonlinear internal wave propagation in horizontally inhomogeneous ocean. Izv. Akad. Nauk SSSR Fiz. Atmos. Okeana, 30, 77-83.

Stanton, T. P., and L. A. Ostrovsky, 1998: Observations of highly nonlinear internal solitons over the continental shelf. Geophys. Res. Lett., 25, 2695-2698.

Turner, J. S., 1973: Buyoancy Effects in Fluids. Cambridge University Press, $367 \mathrm{pp}$.

Vlasenko, V., and K. Hutter, 2002: Numerical experiments of the breaking of internal solitary waves over slope-shelf topography. J. Phys. Oceanogr., 32, 1779-1793.

- L. Ostrovsky, and K. Hutter, 2005a: Adiabatic behavior of strongly nonlinear internal solitary waves in slope-shelf areas. J. Geophys. Res., 110, C04006, doi:10.1029/2004JC002705.

_- N. Stashchuk, and K. Hutter, 2005b: Baroclinic Tides: Theoretical Modeling and Observational Evidence. Cambridge University Press, $350 \mathrm{pp}$.

Whitham, G. B., 1974: Linear and Nonlinear Waves. John Wiley and Sons, $636 \mathrm{pp}$.

Zhou, X., and R. Grimshaw, 1989: The effect of variable currents on internal solitary waves. Dyn. Atmos. Oceans, 14, 17-39. 